\title{
Huge Genetic Inclusion during Conversion of R-Plasmids into MDR Conjugative Plasmids in Multidrug-Resistant Bacteria
}

\author{
Asit Kumar Chakraborty* \\ Department of Biotechnology and Biochemistry, Oriental Institute of Science and Technology, India
}

*Corresponding author: Asit Kumar Chakraborty, Department of Biotechnology and Biochemistry, Oriental Institute of Science and Technology, Vidyasagar University, Midnapore-721102, West Bengal, India

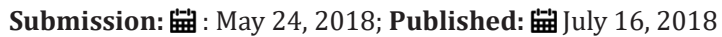

\section{Opinion}

High rate infections and deaths due to multidrug-resistant pathogens have created a horror worldwide. Because MDR bacteria are present in river, sea and air as well as in all household matters, such infections are ubiquitous. Most dangerous step occurred when R-plasmids and integrons (2-9kb) were combined with F'-conjugative plasmid $(50-500 \mathrm{~kb})$ creating MDR conjugative plasmids that highly donated the mdr genes into household pathogens in the environment. Now $40 \%$ of household Escherichia coli and Staphylococcus aureus like Gram (+) and Gram (-) bacteria are ampicillin resistant and also to some extent tetracycline, chloramphenicol, streptomycin, erythromycin, ciprofloxacin, sulfamethoxazole and neomycin resistant.

Before 1600s we do not know that microorganisms (virus, bacteria, fungus and parasites) cause diseases and we blame ghosts and demons. But after the discovery of microscope by Anton Van Leeuwenhoek (1670s) and further pioneering works by Edward Jenner (1790s), Lewis Pasteur (1860s) and David Koch (1880) proved that bacteria were the culprit of many diseases like TB, Cholera, Gonorrhoea and Typhoid. Likely doctor's mind were busy to grow pure bacteria like Vibrio cholerae or Salmonella typhi and find different chemicals that kill it and led to discovery of 5\% phenol as best disinfection as well as $70 \%$ ethanol, reducing surgery infections. Edward Jenner at that time (1789) kicked the concept of vaccination from his pioneering work on Small Pox Vaccination. Thermometer, Stethoscope and many other instruments (X-ray and MRI) were introduced in after 1815 to check the infections without bacterial culture and microscopic view.

Notably, antibiotics are in centre stage of such discoveries since the discovery of penicillin drug by Nobel Laureate Alexander Flaming from slime mold Penicillium notatam in 1928 targeting peptidoglycan cell wall biosynthesis of most Gram (+) and Gram (-) bacteria. Since then hundreds derivatives were made alone for penicillins (ampicillin, cefotaxime and imipenem) for better drug usually called penicillinases resistant drugs. Dr. Selman Waksman discovered over twenty antibiotics that led to Nobel Prize in Physiology or Medicine in 1952. However, such dream could not last long as more potent penicillinases called, oxacillinases, cefotaximases, carbapenem mases were appeared in bacterial plasmids between 1960-1990. Wm Byliss and Ernest Starling introduced Hormone in 1902 discovering secretin that control secretion of pancreatic juice. Never the less, steroid antibiotics have given us some relief as lipid soluble progesterone, entrogens and androgens bind to cytoplasmic receptor and clear the pathogens by eliciting interleukins and cytokines as well as expression of other genes. In other mechanism corticosteroids reduce inflammation.

We know Edward Kendel, Tadeus Reichstein \& Philip Hench (1950), Robert Woodward (1965), Derek Barton \& Add Hassel (1969) and Vladimir Prelog (1975) obtained Nobel for their pioneering work on structure, function and chemical synthesis of steroid hormones which were very effective to cure superbug infection and other infectious diseases. But research indicated that steroids were injurious to health if taken repeatedly.

Interestingly, we also think repeated antibiotic doses are very injurious to health. Because gut bacteria synthesize 20 vitamins (nicotine, thiamine, folate, biotin, riboflavin etc) and other complex signalling molecules (LPS) and also nutrients (butyrate) for intestinal cells those are in tight symbiotic control synthesizing interleukins and cytokines. Save your soul works and mdr genes are created in plasmids and integrons which are first sequenced in 1965 due to advancement of recombinant technology and molecular biology. The amp gene and tet gene were discovered in pBR322 artificially recombined plasmid. The amp gene codes for $286 \mathrm{AA}$ long beta-lactamase enzyme which cleaves lactam A ring of benzylpenicillin where as tet gene codes for $400 \mathrm{AA}$ long trans-membrane proteins that kicks out drug from inside the bacteria into outside . Scientists prepared ampicillin and amoxicillin semi-synthetic drugs that very active on benzyl-penicillin resistant bacteria. But blaTEM, blaOXA, blaSHV could cleave ampicillin and amoxicillin. 
Drug industry was alarmed as drug resistant pathogenesis was apparent in Asia, Africa Europe and America and seems universal in their small plasmid and integron content. So cephalosporin antibiotics were prepared and cefoxitin, ceftriaxone, cefotaxime seem very effective for few years between 1960-1980 but ESBL enzymes were appeared in plasmids which were found $30-70 \mathrm{~kb}$ collecting multiple mdr genes like blaTEM, blaCTX-M and blaOXA-23 including sul1/2, dhfr, catB3, aacC1, aacA1, tet $\mathrm{A} / \mathrm{C}$ and str $\mathrm{A} / \mathrm{B}$. Finer structural analysis indicated that $F^{\prime}$-plasmid $(62.5 \mathrm{~kb})$ was combined with 5-10 mdr genes in presence of many transposons and integrons making a very ideal mdr genes transmission machinery. Sadly, pubmed data analysis indicated that now $>95 \%$ of the isolated bacteria from human were drug resistant and major cases multidrug resistant. This is thus the turning point of MDR spread and horror. The blaNDM-1 beta-lactamase was discovered in 2009 in India and such enzyme can cleave all penicillins, cephallosporins and carbapenems, the wonder drugs that block bacterial cell wall peptidoglycan biosynthesis and we have no cell wall and so less toxic. PenA, PonA and mecA proteins have strong binding capacity to drug and thus inactivate penicillins as also the case of tetM protein which has strong affinity for tetracycline. StrA, strB, aphA2 and aphA4 types enzymes phosphorylate drugs like streptomycin, amikacin, neomycin whereas ANT enzymes adenylate drugs to inactivate it. Large number of acetyl transferases was activated in MDR plasmids like catB3 gene, aacC1 genes and aacA1 genes. Why so many genes are created to perform similar job? Yes, too many new drug derivatives were made and doctors hardly advised poor patients to perform drug sensitivity tests. So first gave penicillin and then tetracycllin, then cefotaxime and then meropenem. So all gut bacteria were dead and no vitamin synthesis. High quality research from US Human Microbiome Project (HMP), European Metagenomics of the Human Intestinal Tract (MetaHIT) and others have demonstrated the beneficial functions of the normal gut flora ( $>35000$ species) on health. At the same time no vitamins were given to patients as well as no probiotics leading to crisis in patients for coenzymes (made from vitamins) needed during all 30,000 biochemical reactions for metabolism and energy production (ATP).

Save your soul works for bacteria and also human cells those are symbiotically controlled. More gene rearrangement as many topoisomerases, recombinases, integrases and ligases are assembled in MDR plasmids. Likely such MDR bacteria are signalled due to secretion of many interleukins and cytokines by intestinal cells that likely facilitated bacteria new gene synthesizing machinery and MDR gene creation. Both bacteria and human saved together by inactivating antibiotics.

All doctors became full this way. Antibiotics over exposure are a criminal offence. Sadly, we see in the poor countries still we use ampicillin and tetracycline and streptomycin increasing TB, syphilis, gonorrhoea, cholera, dysentery and typhoid spread again.
Essentially we also destroyed the utility of vaccination as $1 / 4$ of the unknown genes in large plasmids of MDR bacteria likely create many unknown symbiotic genes.

Acinetobacter baumannii plasmid (accession no. KU549175) has Zeta-toxin, Hemolysin but also one mdr gene ( $\mathrm{mph}$ ), few metal resistant genes (copA, cusA) and thi genes involved in vitamin B1 biosynthesis. Vibrio cholera strain 2012EL-2176 harvoring IncA/ C2 plasmid containing blaCMY-2, blaCTX-M-2, blaTEM-1, floR, aac(3)-IIa , strA/B, sul1/2, dfrA1, dfrA27, tetA, mphA, mdr-genes and also resistant to ciprofloxacin due to mutation in gyrA(S83I)/ parC(S85L) as also seen in plasmid pMRV150 (accession no. EU116442). Bacillus thuringiensis plasmid pBMB293 (Accession no. CP007615, 294kb) has no mdr gene but genes for enterotoxins (protein id. AIM34697), dipterans toxin (protein id. AIM34741) and reverse transcriptase, DNA polymerase $\beta$, DNA topoisomerase III and type II secretion system. Similarly, Bacillus anthrus plasmid pX01 (accession no. CM002399; 171kb) has toxin gene (protein id. AFL55645, 809aa) and in pBMB293 plasmid. Mcr-1 gene has taken colistin super-drug from selves in 2016 as seen in Escherichia coli plasmids, pWJ1 (261kb), pLV23529-MCR-3 (33kb) and pKP37-BE (35kb) (accession nos. KY924928, LT598652, KY964067).

We use 200,000 tons antibiotics per year and so many activities by 7000 million peoples that sea and river are contaminated with drugs (ng- $\mu$ g label) and blaAmp-C gene is induced by low concentration of penicillin drugs and tetO induction is a well known method of expression vector design. In truth, we should blame expression of proteins in bacteria and yeast in biotechnology application may be another culprit of science. Big company, share market, capital market, developed Nations all are for profit and happily long life but all are creating death signals ultimately. Too much pollution for cars, televisions, chemicals, paints, detergents, metals and bacteria heated worse creating MDR genes and antibiotics void. Director General of WHO and UNDP and also Pharmaceutical Scientists are sure that 3-4\% GDP reduction and poverty may be eminent between 2030-2050 if we could not able to discover alternate to antibiotics.

If oral antibiotics would be avoided then such MDR calamity may be delayed. In truth 1943 to 2013 or 70 years we did too much nonsense use of antibiotics. But that is not all, in India, Africa, Latin America; we still prescribe ampicillin and tetracycline to patients as well chickens, pigs, and animals, and also into agricultural land. We absolutely need combined education in school for mandatory mathematics, life science techniques, civic laws and physiology. We absolutely have created this Earth hostile for life. Party-politics, individualistic nations, religions, languages should be combined with humanity. We see phage therapy, enzybiotics, nano-technology, gene-therapy and gene medicines are emerging as profitable pharmaceuticals but question remains as faithful remedies for the control of superbugs. 


\section{(c) (i) Creative Commons Attribution 4.0 International License}

For possible submissions Click Here

Submit Article

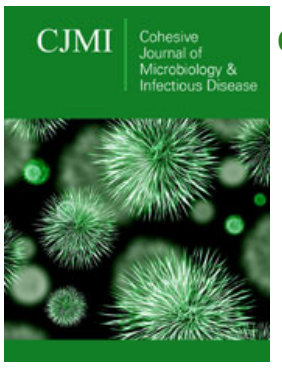

Cohesive Journal of Microbiology \& Infectious Disease Benefits of Publishing with us

- High-level peer review and editorial services

- Freely accessible online immediately upon publication

- Authors retain the copyright to their work

- Licensing it under a Creative Commons license

- Visibility through different online platforms 